Original paper

\title{
Normalized glandular dose coefficients in mammography, digital breast tomosynthesis and dedicated breast CT
}

\author{
A. Sarno ${ }^{\mathrm{a}, \mathrm{b}}$, G. Mettivier ${ }^{\mathrm{a}, \mathrm{b}, *}$, F. Di Lillo ${ }^{\mathrm{a}, \mathrm{b}}$, R.M. Tucciariello ${ }^{\mathrm{a}}$, K. Bliznakova ${ }^{\mathrm{c}}$, P. Russo ${ }^{\mathrm{a}, \mathrm{b}}$ \\ a Dipartimento di Fisica "Ettore Pancini", Università di Napoli Federico II, Napoli, Italy \\ b INFN Sezione di Napoli, Napoli, Italy \\ ${ }^{\mathrm{c}}$ Laboratory of Computer Simulations in Medicine, Technical University of Varna, Varna, Bulgaria
}

\section{A R T I C L E I N F O}

\section{Keywords:}

Breast models

Mean glandular dose

Mammography

Tomosynthesis

Breast CT

\begin{abstract}
A B S T R A C T
Purpose: To provide mean glandular dose (MGD) estimates via Monte Carlo (MC) simulations as a function of the breast models and scan parameters in mammography, digital breast tomosynthesis (DBT) and dedicated breast CT (BCT).

Methods: The MC code was based on GEANT4 toolkit. The simulated compressed breast was either a cylinder with a semi-circular section or ad hoc shaped for oblique view (MLO). In DBT we studied the influence of breast models and exam parameters on the T-factors (i.e. the conversion factor for the calculation of the MGD in DBT from that for a 0-degree projection), and in BCT we investigated the influence on the MGD estimates of the ion chamber volume used for the air kerma measurements.

Results: In mammography, a model representative of a breast undergoing an MLO view exam did not produce substantial differences $(0.4 \%)$ in MGD estimates, when compared to a conventional cranio-caudal (CC) view breast model. The beam half value layer did not present a significant influence on T-factors in DBT $(<0.8 \%)$, while the skin model presented significant influence on MGD estimates (up to $3.3 \%$ at 30 degrees scan angle), increasing for larger scan angles. We derived a correction factor for taking into account the different ion chamber volume used in MGD estimates in BCT.

Conclusions: A series of MC code modules for MGD estimates in 2D and 3D breast imaging have been developed in order to take into account the most recent advances in breast models.
\end{abstract}

\section{Introduction}

Since its proposal in Hammerstein et al [1], the mean glandular dose (MGD) has been adopted as the reference metric for dose assessment in $\mathrm{X}$-ray breast imaging [2]. The MGD in a breast exam can be calculated from measurements of air kerma (mGy) in a fixed area of the field of view and multiplication by suitable conversion coefficients (mGy/ mGy): the normalized glandular dose coefficients (DgN). These coefficients are computed via Monte Carlo (MC) simulations and depend on the breast anatomy, scanner geometry and X-ray spectra characteristics. In MC simulations the scanner geometry is replicated and the shape and composition of the breast are suitably modelled. A common model for breast dosimetry includes an internal breast volume composed of a homogeneous mixture of glandular and adipose tissue, surrounded by an envelope of skin tissue. New 3D breast imaging techniques (dedicated breast computed tomography, BCT) [3] provided a detailed 3D description of the breast anatomy in patients, so permitting more realistic breast models for dosimetry in 2D and 3D X-ray breast imaging [4-9]. In particular, the assumption of a breast skin thickness of 4-5 $\mathrm{mm}$ for the breast model - as commonly adopted in quality assurance protocols [10-13] - was questioned recently, on the basis of measurements obtained by clinical BCT scans $[4,14]$ : they indicated a skin thickness, on average, of $1.45 \mathrm{~mm}$, with no evidence of subcutaneous fat layer [4]. Such a difference in the skin model (4-5 $\mathrm{mm}$ vs. $1.45 \mathrm{~mm}$ ) may affect the dose estimates derived from DgN calculations. In 2D mammography, the MGD estimate is higher for a thinner skin layer $[4,15,16]$. Moreover, in BCT - where higher photon energies than in mammography are adopted - the skin model presents some impact on the (photon energy dependent) MGD estimates adopted for clinical scans [9]. The determination of the 3D dose distribution in the breast volume in BCT can be affected by the breast model, as well [17].

As a further investigative aspect, in quality assurance protocols [11-13] breast models for MGD estimates do not consider the heterogeneous distribution of the breast glandular tissue within the organ

\footnotetext{
* Corresponding author at: Dipartimento di Fisica "Ettore Pancini”, Università di Napoli Federico II, Napoli, Italy.

E-mail address: mettivier@na.infn.it (G. Mettivier).
} 
volume: indeed, they assume that the inner portion of the breast is made of a homogeneous mixture of glandular and adipose tissue. This model simplification may produce a bias in MGD estimates, when compared to estimates based on patient specific structured models derived from clinical BCT scans $[5,6,8,9]$. In particular, this assumption neglects the large variability in the glandular tissue texture and it is representative of a hypothetical "average" breast $[1,8,9]$.

Another point of interest in $\mathrm{DgN}$ calculations for breast dosimetry comes from the observation that the breast models adopted in mammography and DBT are devised for the craniocaudal (CC) view; MGD estimates for mediolateral oblique (MLO) views, for diagnostic views and for particular cases - such as contrast enhanced mammography $[18,19]$ and breasts with silicone inserts [20] - are scarcely addressed in the literature. Some example are the compressed breast model in MLO view presented in Ref. [21] for MGD estimates in DBT and the efforts in refs. [22-24] in defining breast models and dose metrics suitable for partial irradiation spot mammography.

The investigation of patient specific breast models has been addressed by few research groups [25-28]. There is the need to better assess the influence of the texture and spatial location of the glandular tissue on the MGD estimates [8,25,29].

In this scenario, the paradigm for X-ray breast imaging dosimetry is under review. The joint AAPM-EFOMP task group 282 "Development of a new - universal breast dosimetry method" is developing "a new model and corresponding methodology to estimate the breast average glandular dose (AGD) from x-ray based image acquisitions including standard mammography, contrast-enhanced mammography, spot mammography, magnification mammography, and breast tomosynthesis" [30]. It is expected that the investigation in this work may represent a contribution to this task.

In previous papers, we adopted various skin models and investigated the influence of physical processes (bremsstrahlung) and electron cut-off energies adopted in MC simulations for DgN calculation in mammography and BCT $[9,15,31,32]$. In addition, we compared the MDG estimated via homogeneous breast models in mammography and BCT to that obtained with patient specific digital breast phantoms, which reflect the real breast anatomy and shape $[8,9]$.

Taking into account all the previous considerations, we upgraded our MC code for MGD and DgN estimates in 2D and 3D X-ray breast imaging: in this work we present the most recent MC code version by our group which includes the possibility of simulating the geometry adopted in DBT scanners. As a specific additional development included in this code, we calculated the MGD and the conversion coefficients in DBT and introduced a breast model representative of the compressed breast in MLO view. Dose estimates based on the adoption of a MLO breast model in 2D mammography have been produced, as well as simulations showing the influence of the beam quality, skin model and geometrical parameter in DBT, and the influence of the ion chamber volume used in measurements for MGD estimates in BCT.

\section{Materials and methods}

\subsection{Monte Carlo code}

Our Monte Carlo code was based on the GEANT4 toolkit version 10.00 and relied on the low energy physics list Option4 [8,9,15,31,33]. Photoelectric, Compton scatter and Rayleigh photon interactions were simulated (without the inter-atomic interference effects in small angle coherent X-ray scattering [34]). Due to the low influence on the MGD simulations, electrons were not tracked but assumed to release their kinetic energy locally [15]. The composition of the breast glandular, adipose and skin tissues are those reported in Ref. [1]; the pectoralis muscle composition is that in the ICRU 44 report [35]; the compositions of the other materials are those reported in the NIST database. The code was previously validated vs. measurement data [33] and vs. simulated data [15] reported by the AAPM Task Group 195 [36]. However, it was validated exclusively for its use in 2D mammography and BCT dosimetry $[9,15,33]$ : hence, further tests were needed for MGD calculation in a DBT setup. For this reason, we replicated the geometry used in [37] and as well as the simulated data.

The inner part of the irradiated breast was simulated as a homogeneous mixture of adipose and glandular tissue and the MGD was calculated as reported in Ref. [15]:

$\operatorname{MGD}=\frac{\sum_{\mathrm{i}} \mathrm{G}_{\mathrm{i}}(\mathrm{E}) \times \mathrm{E}_{\mathrm{i}}^{\mathrm{dep}}}{\mathrm{f}_{\mathrm{g}} \times \mathrm{W}_{\mathrm{b}}}$

where $E_{\mathrm{i}}^{\mathrm{dep}}$ is the energy released at the interaction event $i, f_{\mathrm{g}}$ is the breast glandular fraction by mass, and $W_{\mathrm{b}}$ is the breast mass (skin excluded). The factor $G(E)$ [38] was evaluated as:

$G(E)=\frac{f_{g} \times \frac{\mu_{\text {en }}}{\rho}(E)_{g}}{f_{g} \times \frac{\mu_{\text {en }}}{\rho}(E)_{g}+\left(1-f_{g}\right) \times \frac{\mu_{\text {en }}}{\rho}(E)_{a}}$

Here, $\mu_{\mathrm{en}} / \rho$ is the mass energy absorption coefficient of glandular (subscript $g$ ) and adipose (subscript $a$ ) tissues, evaluated following the functional interpolation in Ref. [39]. The $G$-factor was evaluated at the energy of the interacting photon. Finally, DgN can be calculated as follows:

$\operatorname{DgN}=\frac{\mathrm{MGD}}{\mathrm{K}}$.

$K$ is the air kerma calculated in a fixed region in the field of view, obtained by scoring the photon fluence passing through a defined part of the irradiated area [31]:

$\mathrm{K}=\sum_{i} \frac{E_{i} \times \frac{\mu_{\mathrm{en}}}{\rho}\left(E_{\mathrm{i}}\right)_{\mathrm{air}}}{S \times \cos \theta_{i}}$

Here, $E_{\mathrm{i}}$ is the energy of the $i$-th photon which passes through a scoring surface of area $S, \mu_{\mathrm{en}} / \rho_{\text {air }}$ is the mass energy absorption coefficient of the (dry) air as contained in the NIST database, $\theta_{i}$ is the angle between the photon direction and the direction perpendicular to the scoring surface. In 2D mammography, the scoring region is usually located at the entrance breast surface $[11-13,15,31,40]$.

In DBT, where the X-ray source rotates over an arc around the breast, the MGD for a single projection, acquired at a scan angle $\alpha$ (MGDa), can be related to the MGD for a 0 degrees projection by means of the t-factor (relative glandular dose, RGD [21]):

$\mathrm{t}=\frac{\mathrm{MGD} \alpha}{\mathrm{MGD}}$

MGD and MGD $\alpha$ are produced with the same tube load (i.e. number of launched photons in MC simulations). $\alpha$ is the angle between the central X-ray beam and the detector plane [21]. The rotation angle around the scanner isocenter $\beta$ is related to $\alpha$ as follows [21]:

$\beta=\alpha+\sin ^{-1}\left(\frac{\operatorname{IDD} \times \sin (\alpha)}{\operatorname{SID}}\right)$.

Here, IDD is the isocenter-to-detector distance and SID is the source-toisocenter distance. Fig. 1a reports a scheme of the irradiation geometry adopted in DBT exams.

The MGD for the DBT exams ( $\mathrm{MGD}_{\mathrm{DBT}}$ ) can be computed by means of the T-factor:

$\mathrm{MGD}_{\mathrm{DBT}}=\mathrm{T} \times \mathrm{K} \times \mathrm{DgN}$.

In Eq. (7), the DgN coefficient is the same as the one adopted for the given breast size and composition and X-ray spectrum in mammography; the air kerma $K$ is measured at 0 degrees projection angle for the total tube load adopted for the considered exam. Then, T-factors $[21,37]$ are derived from $\mathrm{t}$-factors:

$\mathrm{T}=\sum_{\mathrm{i}} \sigma_{\mathrm{i}} \times \mathrm{t}_{\mathrm{i}}$ 


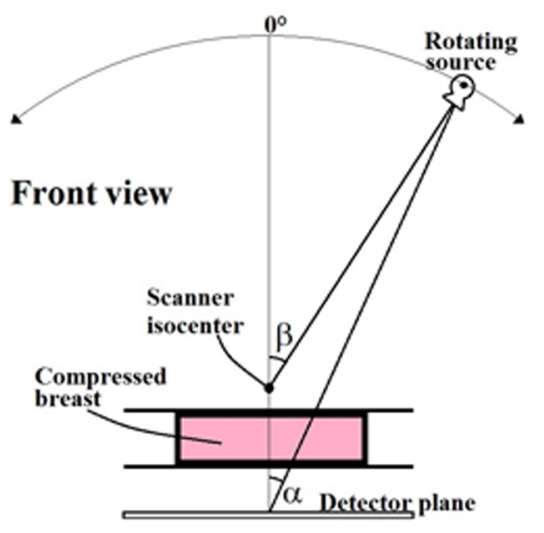

a)

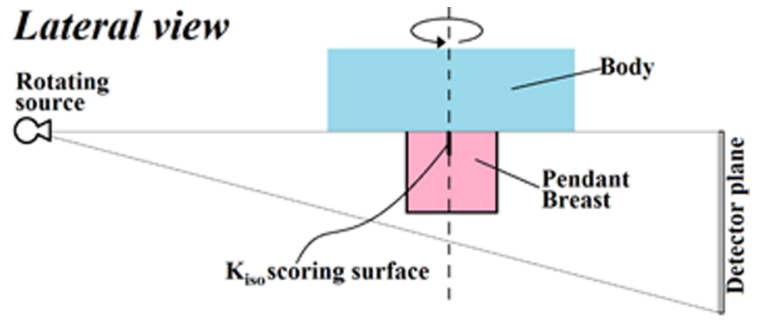

\section{Top view}

b)

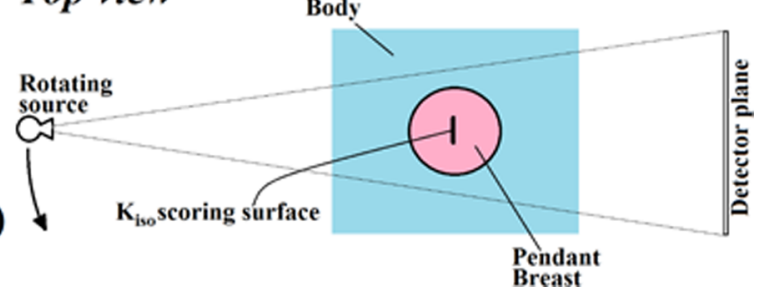

Fig. 1. Scheme of the irradiation geometry in a) a DBT exam and in b) a BCT exam.

with the index $i$ indicating the $\mathrm{i}$-th projection and $\sigma_{\mathrm{i}}$ the partition of the total tube load for the DBT examination among different projections [37].

In BCT, where the uncompressed breast hangs at the scanner isocenter and the gantry rotates over 360 degrees around a vertical axis of rotation, the MGD $\left(\mathrm{MGD}_{\mathrm{BCT}}\right)$ can be calculated from the air kerma measured at the scanner isocenter $\left(\mathrm{K}_{\mathrm{iso}}\right)$ for a full rotation. Hence, MGD $_{\mathrm{BCT}}$ estimates are computed by means of specific $\mathrm{DgN}$ coefficients, $\operatorname{DgN}_{\text {CT }}[9,41-43]$, which take into account the scanner geometry, the $X$ ray spectrum and uncompressed breast shape and composition:

$\mathrm{MGD}_{\mathrm{BCT}}=\mathrm{K}_{\mathrm{ISO}} \times \operatorname{DgN}_{\mathrm{CT}}$

In BCT, the breast is usually simulated as a homogeneous cylinder or a semi-hellipsoid, and its cylindrical symmetry around the axis of rotation permits to simulate the exam with the X-ray source in a fixed position. $\mathrm{K}_{\mathrm{ISO}}$ is computed in the MC simulations as indicated in Eq. (4) from the scored photon fluence passing through a region at the scanner isocenter (Fig. 1b). In the $\mathrm{K}_{\mathrm{ISO}}$ simulations, the patient breast and body are removed from the geometry.

We assessed that the values simulated in this work presented a statistical uncertainty not exceeding $0.2 \%$ of the mean value. It was reached with $10^{8}$ launched photons and a computational time of less than $1 \mathrm{~h}$ at each photon energy.

Data in this work are shown in compliance with the recommendations of the AAPM Task group 268 [44].

\subsection{Breast models and irradiation geometry}

\subsubsection{D mammography and $D B T$}

The breast mass was simulated as a homogenous mixture of adipose and glandular tissue enveloped in a layer simulating the skin. The skin was modelled either as $5.00 \mathrm{~mm}$ of adipose layer (as proposed in Refs. $[37,40]$ ) or as a layer of skin tissue with a thickness of $1.45 \mathrm{~mm}$ (as assumed in [15]): this permitted to compare the results with the two different assumptions on the skin layer composition and thickness. As in Ref. [37], the X-ray point source was isotropic and electronically collimated in order to irradiate exclusively a detector surface of $24 \times 30 \mathrm{~cm}^{2}$ placed at $66.0 \mathrm{~cm}$ from the focal spot of the X-ray tube. The top compression paddle was made of $2.8-\mathrm{mm}$ thick polycarbonate and the support paddle was made of 4.1-mm carbon fiber [37]. The distance between the detector plane and the top of the support paddle was $1.5 \mathrm{~cm}$. The breast in CC view was modelled as a cylinder with a radius of $8.0 \mathrm{~cm}$. In order to explore the impact of simulating the compressed breast in MLO view in mammographic examinations, we replicated the model proposed in Ref. [21]. Here, the breast model included also a portion of the pectoralis muscle, as visible in a mammographic MLO view. The simulation parameters included the glandular fraction by mass, the breast thickness and the chest-to-nipple distance (CND). Fig. 2 shows the scheme of the two compressed breast models. As in Ref. [21] for the DBT exams, we varied the CND value in

\section{Top view}

$\mathrm{CC}$

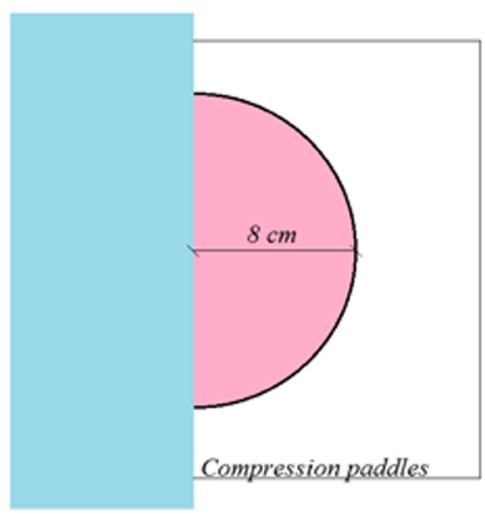

MLO

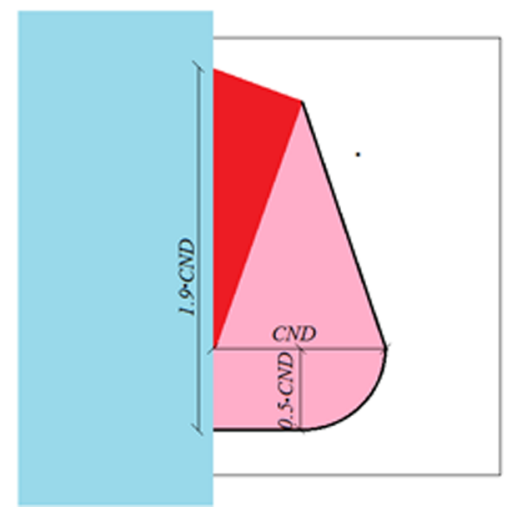

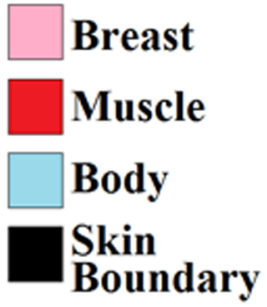

Fig. 2. Top view of the compressed breast models in CC view and MLO view. 
the range $7-13 \mathrm{~cm}$ in order to explore its impact on the MGD estimates in planar mammography. Furthermore, in DBT CC views we investigated the relationship between $\mathrm{T}$ and $\mathrm{t}$ factors and beam HVL, breast characteristics and scanning geometry. For the MGD calculation in mammography, we adopted the Mo/Mo spectrum at $30 \mathrm{kV}$ reported in the TG195 report [36]. In case of DBT simulations, the spectra in Ref. [37] were adopted; the spectra were computed as suggested in Refs. $[45,46]$. In order to vary the beam HVL for a fixed spectrum, the thickness of the inherent filtration was tuned.

\subsubsection{Dedicated breast $C T$}

In order to simulate the BCT irradiation geometry for the calculation of $\mathrm{DgN}_{\mathrm{CT}}$, the pendant breast was modelled as a vertical cylinder enveloped in a $1.45-\mathrm{mm}$ thick layer simulating the skin. The breast was characterized by its diameter, length and glandular fraction by mass. In a previous paper [9] we calculated monoenergetic $\mathrm{DgN}_{\mathrm{CT}}$ coefficients in the energy range $8-80 \mathrm{keV}$ and polyenergetic $\mathrm{DgN}_{\mathrm{CT}}$ coefficients for $49 \mathrm{kV}$ spectra (for $5 \mathrm{HVL}$ levels). In that paper, the conversion coefficients were calculated for a rectangular scoring surface of $18 \times 30 \mathrm{~mm}^{2}$ for determination of the $\mathrm{K}_{\mathrm{ISO}}$, which reflected the cross section of the $6 \mathrm{~cm}^{3}$ ion chamber that we used for $\mathrm{K}_{\mathrm{ISO}}$ measurements. In this work, however, we considered the use of a 100-mm long CT ion chamber for $\mathrm{K}_{\mathrm{ISO}}$ measurements: hence, here we calculated the ion-chamber conversion coefficient, IC, in order to convert those previously-determined $\mathrm{DgN}_{\mathrm{CT}}$ coefficients to the $\mathrm{DgN}_{\mathrm{CT}}$ usable with the new type of ion chamber $\left(\mathrm{DgN}_{\mathrm{CT} 100 \mathrm{~mm}}\right)$ :

$\mathrm{IC}=\frac{\mathrm{K}_{\mathrm{ISO} 6}}{\mathrm{~K}_{\mathrm{ISO} 10}}$

The provision of this IC coefficient allowed us to investigate the influence of the area of the scoring surface on the $\mathrm{DgN}_{\mathrm{CT}}$ values calculated with our MC code. $\mathrm{K}_{\mathrm{ISO}}$ is the simulated air kerma for a $18 \times 30 \mathrm{~mm}^{2}$ photon fluence scoring surface which reflects the cross section of $6 \mathrm{~cm}^{3}$ ion chamber and $\mathrm{K}_{\mathrm{ISO} 10}$ is the simulated air kerma for a $6 \times 100 \mathrm{~mm}^{2}$ scoring area for the photon fluence, which reflects the cross section of a $3 \mathrm{~cm}^{3}$ ion chamber. The $30-\mathrm{mm}$ long and the $100-\mathrm{mm}$ long sides are parallel to the rotation axis of the simulated BCT scanner. Both scoring surfaces were placed at the simulated scanner isocenter. $\mathrm{DgN}_{\mathrm{CT} 100 \mathrm{~mm}}$ was finally computed as follows:

$\operatorname{DgN}_{\mathrm{CT} 100 \mathrm{~mm}}=\mathrm{DgN}_{\mathrm{CT}} \times \mathrm{IC}$

In order to expand the dataset reported in Ref. [9], we computed polyenergetic $\mathrm{DgN}_{\mathrm{CT}}\left(\mathrm{pDgN}_{\mathrm{CT}}\right)$ for the $80 \mathrm{kV}$ X-ray spectrum adopted in Ref. [41] ( $\mathrm{HVL}=5.3 \mathrm{mmAl}$ ). They were calculated from monoenergetic $\mathrm{DgN} \mathrm{N}_{\mathrm{CT}}$ coefficients reported in our previous paper [9]. The spectrum was modelled as suggested in Ref [46]. Data produced in this work were compared to those reported in Ref. [41].

\section{Results}

\subsection{The MGD estimates in MLO mammographic view}

Fig. 3 shows the simulated MGD per photon, in a CC view and in a MLO view in 2D mammography. The CND for MLO view was set in order to produce a breast model with the same glandular mass of the CC view model (with a diameter of $8 \mathrm{~cm}$ ). The distances between the source and the detector and between the source and the top of the compressed breast were in both cases $660 \mathrm{~mm}$ and $595 \mathrm{~mm}$, respectively, with an air gap of $15 \mathrm{~mm}$ between the bottom surface of the breast and the detector plane. Simulations were run for a $30 \mathrm{kV} \mathrm{Mo} / \mathrm{Mo}$ spectrum. The difference in MGD for CC and for MLO views was less than $0.4 \%$.

Fig. 4 shows the simulated MGD per photon for a breast in MLO view, for various CND values in the range $7-13 \mathrm{~cm}$. The MGD is almost constant in the range 7-9 $\mathrm{cm}$ of CND, and it decreases for larger CND values. The maximum difference in the values of MGD per photon is

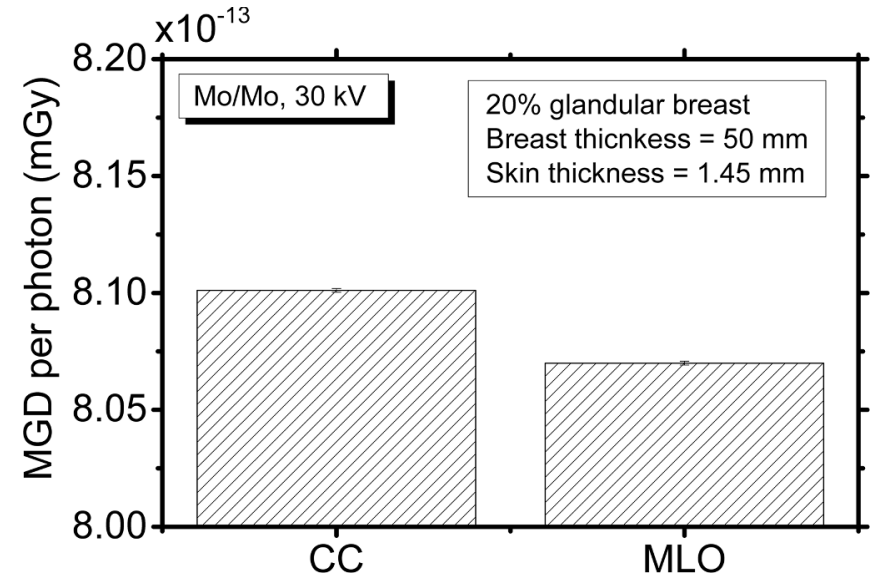

Fig. 3. MGD per photon for a $20 \%$ glandular breast compressed to $50 \mathrm{~mm}$ in CC view and MLO view. The CND in MLO view was tuned in order to have a breast with the same glandular mass in both models. Skin layer $=1.45 \mathrm{~mm}$ skin tissue.

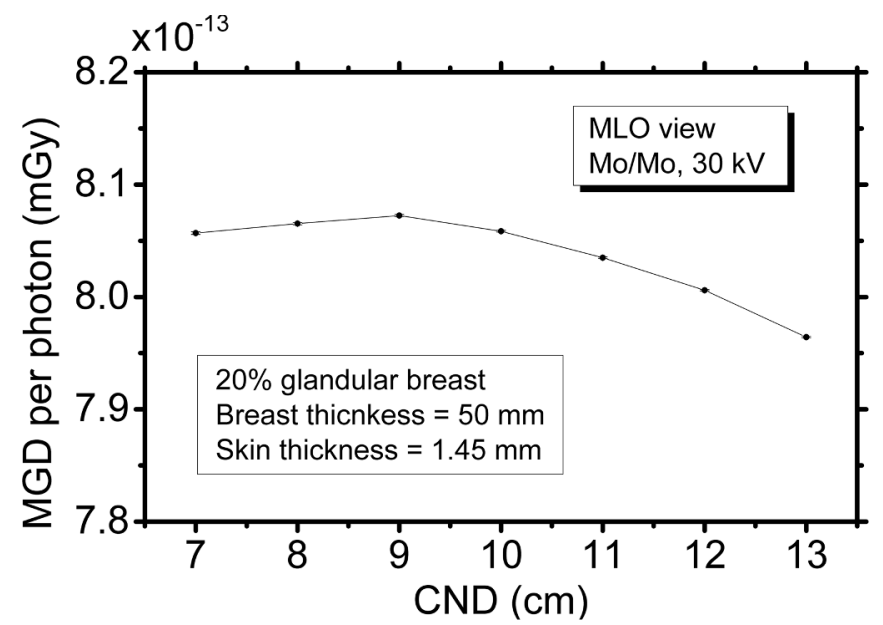

Fig. 4. MGD per photon in MLO view as a function of chest-to-nipple distance. Skin layer $=1.45 \mathrm{~mm}$ skin tissue.

\section{$1.4 \%$ of the maximum value.}

\subsection{Dependence of $t$ and $T$ factors on breast models and scan parameters}

Fig. 5 shows the t-factors calculated with the MC code here adopted and those reported in Ref. [37]. The simulated compressed breast was $50-\mathrm{mm}$ thick with a glandular fraction by mass of $50 \%$. In all cases reported in Fig. 5, the relative differences in t-factor values with respect to the data in Ref. [37] do not exceed $0.4 \%$.

Fig. 6 reports t-factors as a function of projection angle, for four compressed breast thicknesses in the range $20-110 \mathrm{~mm}$. For scan angles less than 15 degrees, the thicker is the breast, the lower is the corresponding t-factor. For greater scan angles, no monotonic dependence between t-factors and breast thickness was observed. For the thickest breasts $(80 \mathrm{~mm}$ and $110 \mathrm{~mm})$ and for scan angles lower than 20 degrees, $\mathrm{t}$-factor differences are negligible; for higher scan degrees, $\mathrm{t}$ factors for $110-\mathrm{mm}$ thick breast resulted higher than those for $80-\mathrm{mm}$ thick breasts. Modelling the skin envelope as a $1.45-\mathrm{mm}$ thick skin tissue layer (instead of $5 \mathrm{~mm}$ adipose tissue) increases the t-factors (Fig. 7, $34 \mathrm{kV} \mathrm{W/Rh}$ and $29 \mathrm{kV} \mathrm{Rh} / \mathrm{Rh}$ spectra, $50 \mathrm{~mm}$ breast thickness). The differences are greater for increasing scan angles. For instance, for $29 \mathrm{kV} \mathrm{Rh} / \mathrm{Rh}$ spectrum, for a 15 degrees scan angle the former model produced a t-factor $0.5 \%$ larger than the one obtained with a $5 \mathrm{~mm}$ adipose envelope; this relative difference increased to $3.3 \%$ for a projection angle of 30 degrees. 


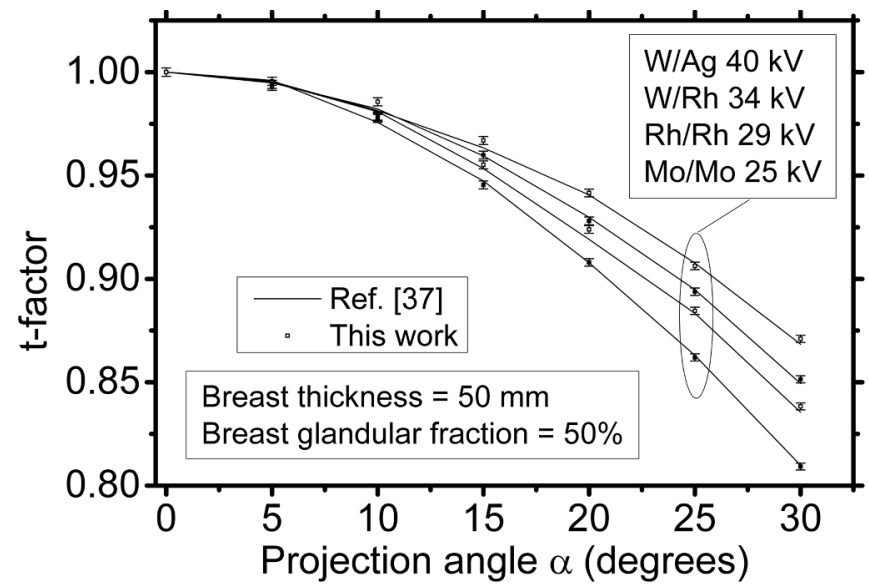

Fig. 5. Comparison between t-factors reported in Ref. [37] and those computed with the code adopted in this work. SID $=620 \mathrm{~mm}$; IDD $=40 \mathrm{~mm}$. Skin layer $=5 \mathrm{~mm}$ adipose tissue.

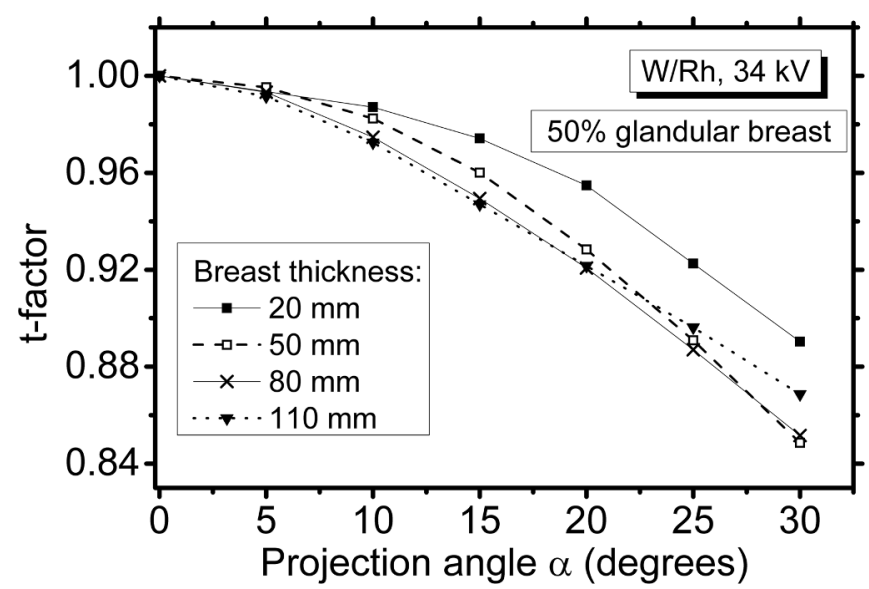

Fig. 6. t-factors for various compressed breast thicknesses. SID $=620 \mathrm{~mm}$; IDD $=40 \mathrm{~mm}$. Skin layer $=5 \mathrm{~mm}$ adipose tissue.

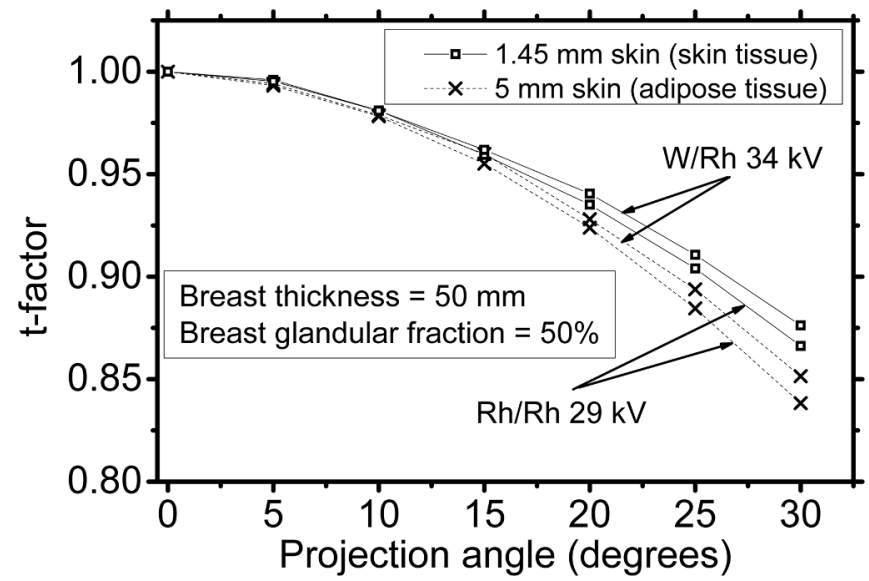

Fig. 7. t-factors for the skin layer simulated either as $5 \mathrm{~mm}$ of adipose tissue or $1.45 \mathrm{~mm}$ layer made of skin tissue. SID $=620 \mathrm{~mm}$; IDD $=40 \mathrm{~mm}$.

For fixed tube voltage and anode filter combination, the HVL has little influence on the T-factor (Fig. 8). For $29 \mathrm{kV} \mathrm{Rh/Rh} \mathrm{spectra} \mathrm{and} \mathrm{a}$ $30 \mathrm{~mm}$ compressed breast, using a spectrum with an HVL of 0.410 $\mathrm{mmAl}$ instead of $0.337 \mathrm{mmAl}$ increased the T-factor by $0.8 \%$ (Fig. 8a). The maximum deviation among the T-factors computed for $34 \mathrm{~W} / \mathrm{Rh}$ spectra and $50 \mathrm{~mm}$ breast thickness does not exceed $0.3 \%$ (Fig. $8 \mathrm{~b}$ ).
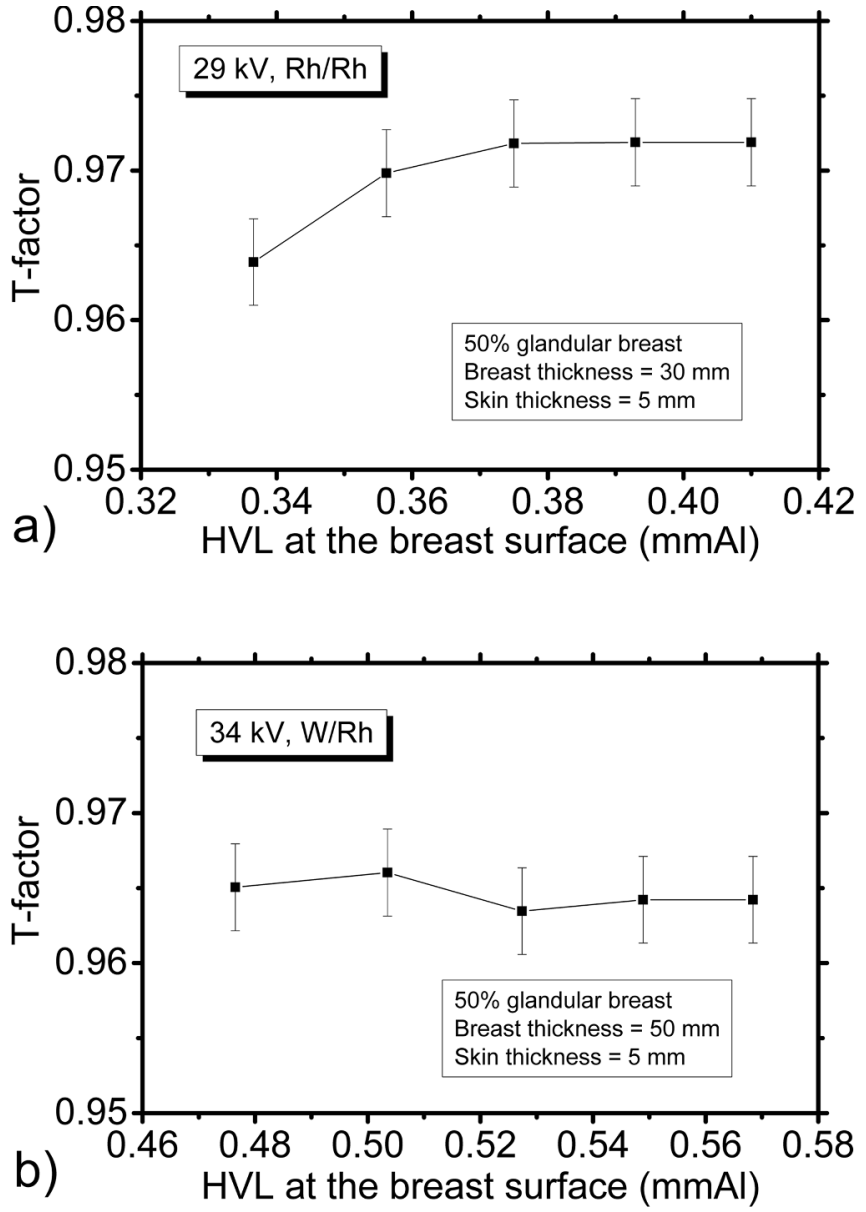

Fig. 8. T-factors at varying HVL beam values for a) $30 \mathrm{~mm}$ breast thickness and $29 \mathrm{kV} \mathrm{Rh} / \mathrm{Rh}$ beam spectrum and b) $50 \mathrm{~mm}$ breast thickness and $34 \mathrm{kV} \mathrm{Rh} / \mathrm{Rh}$ beam spectrum. SID $=650 \mathrm{~mm}$; IDD $=47 \mathrm{~mm}$. 25 projections over 50 degrees scan angle. Skin layer $=5 \mathrm{~mm}$ adipose tissue.

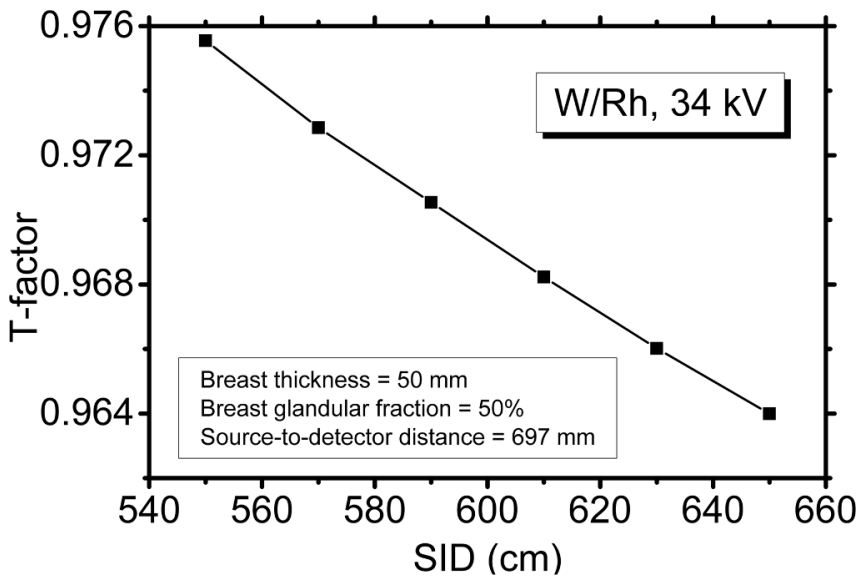

Fig. 9. T-factors at source-to-isocenter distances. The source to isocenter distance was fixed at $697 \mathrm{~mm}$. 25 projections over 50 degrees scan angle. Skin layer $=5 \mathrm{~mm}$ adipose tissue.

Fig. 9 shows the T-factors at various source-to-isocenter distances. For fixed breast characteristics and source-to-detector distance, the T-factor decreased by increasing the SID; increasing the SID from $55 \mathrm{~cm}$ to $65 \mathrm{~cm}$ reduced the T-factor by $1.2 \%$. 


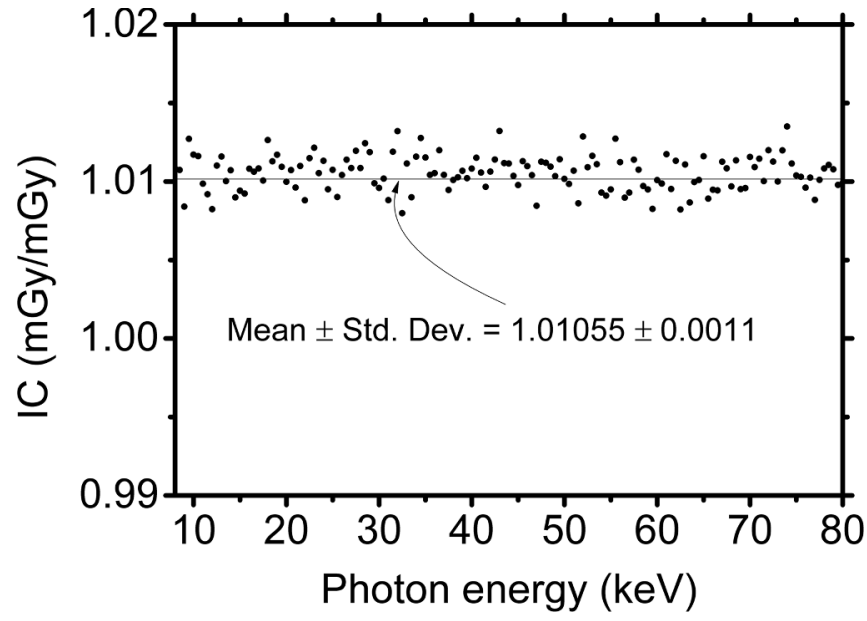

Fig. 10. Conversion factor between $\mathrm{DgN}_{\mathrm{CT}}$ calculated for a $6 \mathrm{~cm}^{3}$ ion chamber and the one calculated for air kerma measured by means of a 100-mm CT ion chamber.

\subsection{IC conversion factor in $B C T$}

Fig. 10 shows conversion factors IC for deriving $\mathrm{DgN}_{\mathrm{CT} 100 \mathrm{~mm}}$ from those in Ref. [9]. These last are computed for a $6 \mathrm{~cm}^{3}$ ion chamber (for air kerma determinations) with a cross section of $18 \times 30 \mathrm{~mm}^{2}$. IC coefficients were computed for photon energies between $8 \mathrm{keV}$ and $80 \mathrm{keV}$. Since the difference is due to the sole geometrical configuration of the scoring region, the coefficients are not expected to depend on the $\mathrm{X}$-ray beam energy. The average IC value was 1.01 , indicating that there is a $1 \%$ increase in the estimate of $\mathrm{K}_{\mathrm{ISO}}$ when changing the scoring area from $6 \times 100 \mathrm{~mm}^{2}$ to $18 \times 30 \mathrm{~mm}^{2}$, an effect due to the divergence of the X-ray beam and consequent variation of the air kerma in different positions in the isocenter irradiation plane. Due to its independence on the beam energy, this value can be also used for polyenergetic $\mathrm{DgN}_{\mathrm{CT}}$ coefficients, when changing the $\mathrm{K}_{\mathrm{ISO}}$ scoring surface area between the two values $\left(18 \times 30 \mathrm{~mm}^{2}\right.$ and $\left.6 \times 100 \mathrm{~mm}^{2}\right)$.

Fig. 11 shows $\mathrm{pDgN}_{\mathrm{CT}}$ values for a $80 \mathrm{kV} \mathrm{W/Cu}$ spectrum. They are calculated for a cylindrical breast model with a length equal to 1.5 times its radius. Data in Fig. 11, together with those calculated for a breast with length of 1 or 2 times the breast radius, are reported in the Supplementary material. Data reported in Ref. [41] (calculated for 50\% glandular breast) are also reported in Fig. 11, for comparison. The latter data are $7 \%$ higher for the case of an $8-\mathrm{cm}$ breast diameter and the

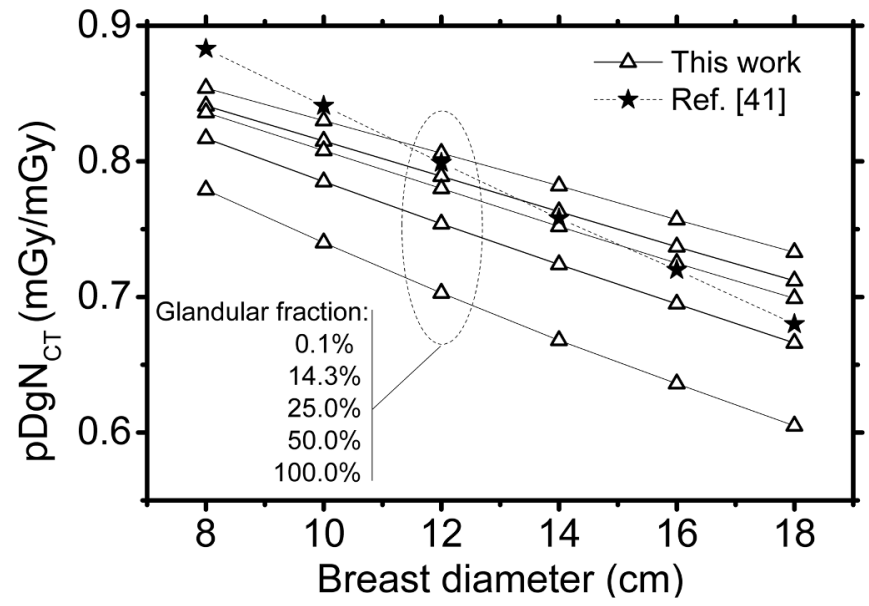

Fig. 11. Polyenergetic $\mathrm{DgN}_{\mathrm{CT}}$ for $\mathrm{W} / \mathrm{Cu}$ spectrum at $80 \mathrm{kV}, \mathrm{HVL}=5.3 \mathrm{mmAl}$. Breast length $=1.5 \times$ breast radius. For comparison, data in Ref. [41] for $50 \%$ glandular breast are shown. difference decreases as the breast diameter increases, down to a difference of $2 \%$ for a $18-\mathrm{cm}$ breast diameter (Fig. 11).

Supplementary data associated with this article can be found online at https://doi.org/10.1016/j.ejmp.2018.09.002.

\section{Discussion}

In order to contribute to the process of reviewing the paradigm in Xray breast imaging dosimetry, we developed a Monte Carlo code for assessing normalized glandular dose coefficients in 2D and 3D breast dosimetry. In this paper, we presented the most recent version of this code, including the added features for computing the MGD in DBT and in MLO views. We replicated the compressed breast model proposed in Ref. [21] for representing the breast in MLO mammographic view, which comprises a portion of the pectoral muscle. Using the MC estimates, we provided indication that adopting a suitable breast model for such a view does not produce large differences in MGD compared to that obtained for a conventional compressed breast model adopted for representing the organ in CC view. The difference for the simulated $\mathrm{Mo} / \mathrm{Mo}$ spectrum at $30 \mathrm{kV}$, and a $50 \mathrm{~mm}$ thick and $50 \%$ glandular breast, was about $0.4 \%$. As already shown in mammography $[4,15,16]$ and in BCT [9], the specific skin model adopted may have a significant impact on MGD estimates in DBT. Here we compared, for two X-ray spectra, the differences in the computed t-factors by adopting a $1.45 \mathrm{~mm}$ of skin layer instead of a $5 \mathrm{~mm}$ adipose layer, as envisaged in current quality assurance protocols. In the performed simulations, a $1.45-\mathrm{mm}$ thick skin layer produced higher $\mathrm{t}$-factors, for any scan angle in the range 0-30 degrees. However, the larger is the scan angle, the larger is the influence of the skin model on MGD estimates. For a $50 \mathrm{~mm}$ breast thickness and $50 \%$ glandular fraction by mass, and for a $29 \mathrm{kV}$ $\mathrm{Rh} / \mathrm{Rh}$ spectra and 15 degrees scan angle, a 1.45-mm thick skin layer led to t-value $0.5 \%$ higher than that for $5 \mathrm{~mm}$ adipose layer; such a difference increased to $3.3 \%$ for a scan angle of 30 degrees in a DBT scan. When all the other parameter are fixed, varying the beam HVL did not produce any significant difference in T-factors.

In a previous work [9], we calculated the $\mathrm{DgN}_{\mathrm{CT}}$ for MGD estimates in BCT. There, a $18 \times 30 \mathrm{~mm}^{2}$ scoring surface for the air kerma calculation was adopted: this choice pointed to using an ion chamber of $6 \mathrm{~cm}^{3}$ sensitive volume at the scanner isocenter, for measuring the air kerma (as required in Eq. (3)). On the other hand, when using a $100 \mathrm{~mm}$ CT ion chamber for such a measurement, the scoring surface changes (to $6 \times 100 \mathrm{~mm}^{2}$ ). In order to calculate the corresponding new $\mathrm{DgN} \mathrm{N}_{\mathrm{CT}}$ coefficients, we have calculated an (energy independent) correction factor IC which is the ratio of the two simulated $\mathrm{K}_{\mathrm{ISO}}$ values. This IC ratio is expected to be slightly greater than 1 since the 100 -mm long ion chamber extends at the axis of rotation at larger cone angles than the 6$\mathrm{cm}^{3}$ chamber, where the air kerma decreases due to beam divergence. Its value (IC $=1.01$ as calculated with our MC code) can be used for the case of either monoenergetic or polyenergetic X-ray beams in order to convert $\mathrm{DgN}_{\mathrm{CT}}$ values obtained with either ion chambers for $\mathrm{K}_{\mathrm{ISO}}$ measurements. Polyenergetic $\mathrm{DgN}_{\mathrm{CT}}$ were calculated for an $80 \mathrm{kV}$ spectrum (adopted in Ref. [41]) and are reported in the Supplementary material in tabular form. The $\mathrm{DgN}_{\mathrm{CT}}$ values calculated with the MC code in this work showed differences between $2 \%$ and $7 \%$ from the values reported in Ref. [41].

\section{Conclusions}

We presented the most recent version of our Monte Carlo code for the MGD calculation in 2D and 3D X-ray breast imaging. It comprises also a module for the t-factors and T-factors calculation in DBT. The code was validated vs. literature data reported in Ref. [37]. We developed a compressed breast model for representing the breast undergoing a mammographic exam in MLO view. The MGD calculated with such a model did not significantly differ from that calculated for the model of the breast undergoing a CC view exam. The skin model in DBT 
presented a significant influence on the calculated t-factor. A breast with a $1.45 \mathrm{~mm}$ skin produced higher $\mathrm{t}$-factors than that with a skin simulated as a $5 \mathrm{~mm}$ adipose tissue, at any scan angle. The larger is the scan angle, the larger is the difference. Finally, we computed a conversion factor for the use of $\mathrm{DgN}_{\mathrm{CT}}$ coefficients reported in Ref. [9] in conjunction with air kerma measurements by means of $100 \mathrm{~mm}$ CT ion chamber. Polyenergetic $\mathrm{DgN}_{\mathrm{CT}}$ coefficients were calculated with the new breast model, for the $80 \mathrm{kV}$ spectrum adopted by a clinical BCT scanner developed at UC Davis.

\section{Acknowledgements}

The authors would like to thank prof. D. R. Dance for sharing data of the Ref. [37] reported in Fig. 5. This work was supported by INFN and by the MAXIMA project: Three dimensional breast cancer models for Xray imaging research. This project has received funding from the European Union's Horizon 2020 research and innovation programme under grant agreement No. 692097.

\section{References}

[1] Hammerstein RG, Miller DW, White DR, Masterson ME, Woodard HQ, Laughlin JS. Absorbed radiation dose in mammography. Radiology 1979;130:485-91.

[2] Dance DR, Sechopoulos I. Dosimetry in X-ray-based breast imaging. Phys Med Biol 2016;61:R271-304.

[3] Sarno A, Mettivier G, Russo P. Dedicated breast computed tomography: basic aspects. Med Phys 2015;42:2786-804.

[4] Huang S-Y, Boone JM, Yang K, Kwan ALC, Packard NJ. The effect of skin thickness determined using breast CT on mammographic dosimetry. Med Phys 2008;35:1199-206.

[5] Sechopoulos I, Bliznakova K, Qin X, Fei B, Feng SSJ. Characterization of the homogeneous tissue mixture approximation in breast imaging dosimetry. Med Phys 2012;39:5050-9.

[6] Hernandez AM, Seibert JA, Boone JM. Breast dose in mammography is about 30\% lower when realistic heterogeneous glandular distributions are considered. Med Phys 2015;42:6337-48.

[7] Boone JM, Hernandez AM, Seibert JA. Two-dimensional breast dosimetry improved using three-dimensional breast image data. Radiol Phys Technol 2017;10:129-41.

[8] Sarno A, Mettivier G, Di Lillo F, Bliznakova K, Sechopoulos I, Russo P. Homogeneous vs. patient specific breast models for Monte Carlo evaluation of mean glandular dose in mammography. Phys Med 2018;51:56-63.

[9] Sarno A, Mettivier G, Tucciariello RM, Bliznakova K, Boone JM, Sechopoulos I, et al. Monte Carlo evaluation of glandular dose in cone-beam X-ray computed tomography dedicated to the breast: Homogeneous and heterogeneous breast models. Phys Med 2018;51:99-107.

[10] Hendrick RE, Bassett L, Botsco MA, Deibel D, Feig S, Gray J. Mammography quality control manual. Reston, Va: American College of Radiology; 1999.

[11] Institute of Physics and Engineering in Medicine (IPEM). The commissioning and routine testing of mammographic X-ray systems. IPEM Report 89 (York, United Kingdom: IPEM); 2005.

[12] European Commission 2006 4th edition of the European Guidelines for Quality Assurance in Breast Cancer Screening and Diagnosis.

[13] ACR. Digital mammography quality control Manual; 2016.

[14] Shi L, Vedantham S, Karellas A, O'Connell AM. Technical Note: Skin thickness measurements using high-resolution flat-panel cone-beam dedicated breast CT. Med Phys 2013;40. 031913-1-6.

[15] Sarno A, Mettivier G, Di Lillo F, Russo P. A Monte Carlo study of monoenergetic and polyenergetic normalized glandular dose (DgN) coefficients in mammography. Phys Med Biol 2016;62:306-25.

[16] Massera RT, Tomal A. Skin models and their impact on mean glandular dose in mammography. Phys Med 2018;51:38-47.

[17] Mettivier G, Costa M, Lanconelli N, Ianiro A, Pugliese M, Quarto M, et al. Evaluation of dose homogeneity in cone-beam breast computed tomography. Radiat Prot Dosim 2017:175:473-81.

[18] Dance DR, Young KC. Estimation of mean glandular dose for contrast enhanced digital mammography: factors for use with the UK, European and IAEA breast dosimetry protocols. Phys Med Biol 2014;59:2127-37.

[19] Tzamicha E, Yakoumakis E, Tsalafoutas IA, Dimitriadis A, Georgiou E, Tsapaki V, et al. Dual-energy contrast-enhanced digital mammography: Glandular dose estimation using a Monte Carlo code and voxel phantom. Phys Med 2015;31:785-91.
[20] Daskalaki A, Bliznakova K, Pallikarakis N. Evaluation of the effect of silicone breast inserts on X-ray mammography and breast tomosynthesis images: a Monte Carlo simulation study. Phys Med 2016;32:353-61.

[21] Sechopoulos I, Suryanarayanan S, Vedantham S, D'Orsi C, Karellas A. Computation of the glandular radiation dose in digital tomosynthesis of the breast. Med Phys 2007;34:221-32.

[22] Liu B, Goodsitt M, Chan HP. Normalized average glandular dose in magnification mammography. Radiology 1995;197:27-32.

[23] Koutalonis M, Delis H, Spyrou G, Costaridou L, Tzanakos G, Panayiotakis G. Monte Carlo generated conversion factors for the estimation of average glandular dose in contact and magnification mammography. Phys Med Biol 2006;51:5539-48.

[24] Sarno A, Dance DR, van Engen RE, Young KC, Russo P, Di Lillo F, et al. A Monte Carlo model for mean glandular dose evaluation in spot compression mammography. Med Phys 2017;44:3848-60.

[25] Dance DR, Hunt RA, Bakic PR, Maidment ADA, Sandborg M, Ullman G, et al. Breast dosimetry using high-resolution voxel phantoms. Radiat Prot Dos 2005;114:359-63.

[26] Wang W, Qiu R, Ren L, Liu H, Wu Z, Li C, et al. Monte Carlo calculation of conversion coefficients for dose estimation in mammography based on a $3 \mathrm{D}$ detailed breast model. Med Phys 2017;44:2503-14.

[27] Mettivier G, Bliznakova K, Sechopoulos I, Boone JM, Di Lillo F, Sarno A, et al. Evaluation of the BreastSimulator software platform for breast tomography. Phys Med Biol 2017;62:6446-66.

[28] Baneva Y, Bliznakova K, Cockmartin L, Marinov S, Buliev I, Mettivier G, et al. Evaluation of a breast software model for 2D and 3D X-ray imaging studies of the breast. Phys Med 2017;41:78-86.

[29] Geeraert N, Klausz R, Muller S, Bloch I, Bosmans H. Evaluation of exposure in mammography: limitations of average glandular dose and proposal of a new quantity. Radiat Prot Dos 2015;165:342-5.

[30] AAPM 282 - website: https://www.aapm.org/org/structure/?committee code = TG282 [accessed on $06 / 27 / 2018$ ].

[31] Sarno A, Mettivier G, Russo P. Air kerma calculation in Monte Carlo simulations for deriving normalized glandular dose coefficients in mammography. Phys Med Biol 2017;62:N337-49.

[32] Mettivier G, Fedon C, Di Lillo F, Longo R, Sarno A, Tromba G, et al. Glandular dose in breast computed tomography with synchrotron radiation. Phys Med Biol 2016;61:569-87.

[33] Sarno A, Masi M, Antonelli N, Di Lillo F, Mettivier G, Castriconi R, et al. dose volume distribution in digital breast tomosynthesis: a phantom study. IEEE Trans Rad Pl Med Sc 2017;1:322-8.

[34] Paternò G, Cardarelli P, Contillo A, Gambaccini M, Taibi. Geant4 implementation of inter-atomic interference effect in small-angle coherent X-ray scattering for materials of medical interest. Phys Med 2018;51:64-70.

[35] International Commission on Radiation Units and Measurements. Tissue substitutes in radiation dosimetry and measurement. Bethesda, Md.: International Commission on Radiation Units and Measurements; 1989.

[36] Sechopoulos I, Ali ES, Badal A, Badano A, Boone JM, Kyprianou IS, et al. Monte Carlo reference data sets for imaging research: executive summary of the report of AAPM Research Committee Task Group 195. Med Phys 2015;42:5679-91.

[37] Dance DR, Young KC, Van Engen RE. Estimation of mean glandular dose for breast tomosynthesis: factors for use with the UK, European and IAEA breast dosimetry protocols. Phys Med Biol 2010;56:453-71.

[38] Boone JM. Glandular breast dose for monoenergetic and high-energy X-ray beams: Monte Carlo assessment. Radiology 1999;213:23-37.

[39] Fedon C, Longo F, Mettivier G, Longo R. GEANT4 for breast dosimetry: parameters optimization study. Phys Med Biol 2015;60:N311-23.

[40] Dance DR. Monte-Carlo calculation of conversion factors for the estimation of mean glandular breast dose. Phys Med Biol 1990;35:1211-9.

[41] Boone JM, Shah N, Nelson TR. A comprehensive analysis of DgNCT coefficients for pendant-geometry cone-beam breast computed tomography. Med Phys 2004;31:226-35.

[42] Thacker SC, Glick SJ. Normalized glandular dose (DgN) coefficients for flat-panel CT breast imaging. Phys Med Biol 2004;49:5433-44.

[43] Sechopoulos I, Feng SSJ, D’Orsi CJ. Dosimetric characterization of a dedicated breast computed tomography clinical prototype. Med Phys 2010;37:4110-20.

[44] Sechopoulos I, Rogers DW, Bazalova-Carter M, Bolch WE, Heath EC, McNitt-Gray MF, et al. RECORDS: improved reporting of montE CarlO RaDiation transport studies: report of the AAPM Research Committee Task Group 268. Med Phys 2018;45:e1-5.

[45] Boone JM, Fewell TR, Jennings RJ. Molybdenum, rhodium, and tungsten anode spectral models using interpolating polynomials with application to mammography. Med Phys 1997;24:1883-11874.

[46] Hernandez AM, Boone JM. Tungsten anode spectral model using interpolating cubic splines: Unfiltered x-ray spectra from $20 \mathrm{kV}$ to $640 \mathrm{kV}$. Med Phys 2014;41. 042101 1-15. 